\title{
Clustering of Brain Tumors in Brain MRI Images Based on Extraction of Textural and Statistical Features
}

\author{
https://doi.org/10.3991/ijoe.v16i12.16929 \\ Hamed Samadi Goushchi, Yaghoub Pourasad ${ }^{(\varpi)}$ \\ Urmia university of Technology, Urmia, Iran \\ alireza.e.amiriegmail.com
}

\begin{abstract}
The purpose of this article is to investigate techniques for classifying tumor grade from magnetic resonance imaging (MRI). This requires early diagnosis of the brain tumor and its grade. Magnetic resonance imaging may show a clear tumor in the brain, but doctors need to measure the tumor in order to treat more or to advance treatment. For this purpose, digital imaging techniques along with machine learning can help to quickly identify tumors and also treatments and types of surgery. These combined techniques in understanding medical images for researchers are an important tool to increase the accuracy of diagnosis. In this paper, methods used to classify brain tumors, including preprocessing, screening, tissue extraction, and statistical features of the tumor using two types of T1W and Flair brain MRI images and also the method of dimensionality reduction of extracted features and how to train them in classification are also explained. Determine the tumor area using three classification of Fuzzy Logic C-Means Clustering (FCM), Probabilistic Neural Networks (PNN) and Support Vector Machines (SVM). In this paper, simulated and real MRI images are used. The results obtained from the proposed methods in this paper are compared with the reference results and the results show that the proposed approach can increase the reliability of brain tumor diagnosis.
\end{abstract}

Keywords-Brain Tumor, MRI images, Classification, Fuzzy Logic C-Means Clustering (FCM), Probabilistic Neural Networks (PNN), Support Vector Machines (SVM).

\section{Introduction}

MRI has attracted considerable attention in the medical engineering community because it has been a noninvasive diagnostic technique and its importance is clearly evident in medical applications [1-3]. An approach for segmentation of brain tumors from FLAIR MRI, using Fully Convolutional Neural Networks (FCNNs), has been proposed in [4]. The FCNNs are trained in 25625256 packs that consist only of subregions of the primary images that contain the tumor and can provide segmentation of full-size FLAIR MRI scans, which helps Radiologists improve their diagnosis. In [5], an adaptive threshold value selection technique, with morphological activity, has been proposed to identify brain tumors. The proposed method can match different types of 
brightness values of pixels of MRI FLAIR images and efficiently detect the tumor. In [6] proposed a wavelet deformation for tumor diagnosis and classification. Low-level features can effectively describe the image, but they are low-level and their leadership is limited because different types of brain tumors have a similar appearance (such as border, texture, size, and shape). In [7], they proposed a wavelet-based method for extracting features from MR images. In this method, segmentation of brain MR images was performed using the Markov Random Field (MRF) model. Medical imaging techniques (such as Computed Tomography (CT), Positron Emission Tomography (PET) and Magnetic Resonance Imaging (MRI) are used to diagnose brain tumors [8]. In [9], brain tumor recovery was suggested using FV and uses the space pyramid matching, divides images into rectangular grids, computes BoW descriptors for each grid and finally, the results were weighted and combined. According to previous research, more accurate, computer-aided diagnosis (CAD) studies are needed to study and interpret images. Many automated classification techniques have been proposed for MRI classification (normal / abnormal) in order to improve classification time and reduce human error. In this article, different techniques for MR image classification are discussed in which various tools are used to extract features and classification. Segmentation of MRI images using convolutional neural networks is presented in article [10]. In [11 and 12] proposed a fully automatic system for detecting slices that include tumor and, to distinguish the tumor area with numerical and experimental manner. From the results, it is clear that tumor identification, in MR images, is predictable using Convolutional Neural Network (CNN) and the efficacy of this prediction is appropriate.

\section{Research Method}

\subsection{Classifier system}

Probabilistic Neural Network classifier (PNN): The probabilistic neural network classifier is a hybrid classifier that combines the two perspectives of non-parametric estimators of probability density functions and Bayesian classification [13]. The PNN classifier has a decision function as follows:

$$
d_{k}(x)=\frac{1}{(2 \pi)^{\frac{n}{2}} \sigma^{n} N_{k}} \sum_{i=1}^{N_{k}} e^{\frac{-\left\|x-x_{i}\right\|^{2}}{2 \sigma^{2}}}
$$

Where, n: The number of textural features of the input pattern; Nk: Number of attributes related to Xk; X: Experimental feature sampler that is classified; xi: i Main test input feature; $\sigma_{\mathrm{a}}$ regulator parameter with a value between zero and one.

From this relation, it can be concluded that if the fraction form $\left(\left\|x_{i}-x\right\|_{)}\right.$increases in the exponential function, the function tends to zero, and as a result, the two attribute vectors have little similarity, but if the fraction form $\left(\left\|x_{i}-x\right\|_{\text {) decreases in the }}\right.$ 
exponential function, the function tends to one desire. As a result, the two feature vectors are very similar.

The PNN classifier structure consists of 4 layers as shown in Figure 1. The input, the pattern, the summation, and the output layers are the constituent layers of the PNN classification.

Support Vector Machine (SVM) classifier: In machine learning, SVM is an algorithm with an observer that is responsible for data analysis and is one of the classification methods. Sometimes used for regression issues. In the mentioned algorithm, the input data are in the form of points in space and are divided into two categories and placed in the next $n$ space on the data scatter diagram. The backup vector machine forms a superplate or set of superplanes in a high-dimensional space that is used for classification. A good separation can be achieved using the hyperplane that is farthest from the nearest training data in each category. Reducing the generalization error of the classifier depends on increasing the margin.

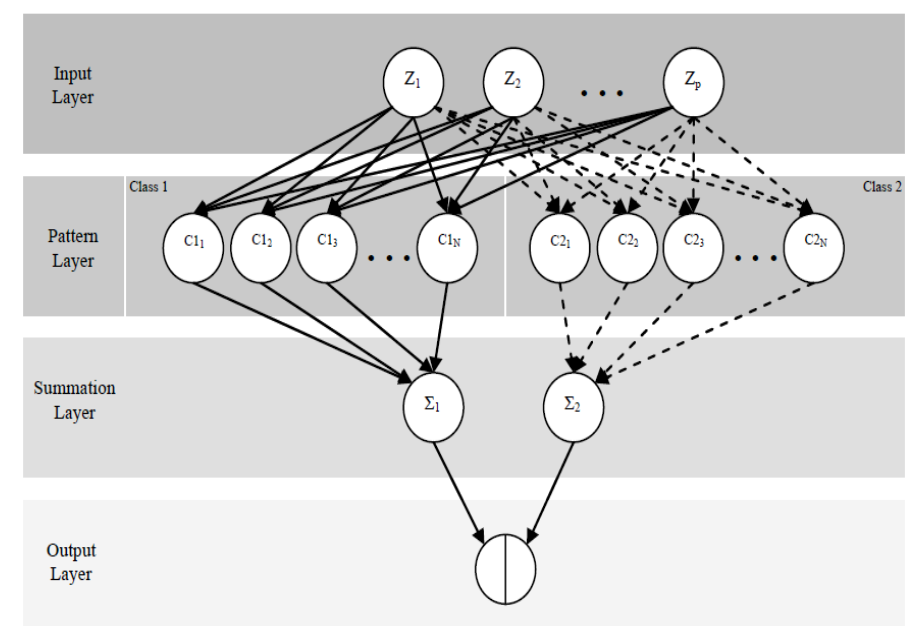

Fig. 1. The schematic of PNN classifier structure

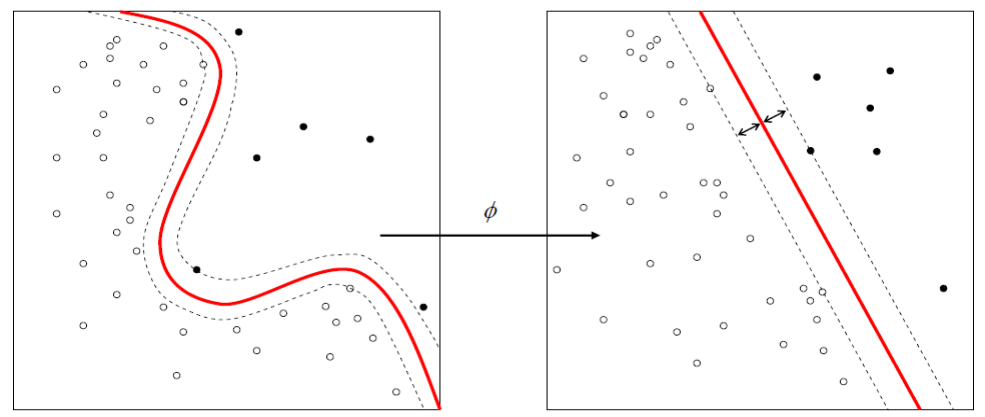

Fig. 2. Feature space mapping in SVM classifier 
Mean-C Fuzzy Logic (FCM) classifier: This classifier uses the neighborhood location information of each pixel. Unlike some classifications, such as the k-means algorithm, which assigns each pixel to a category, the FCM algorithm allows each pixel to be associated with multiple categories with varying degrees of membership.

The FCM algorithm attempts to partition a finite collection of $n$ \{ \displaystyle $n\} n$ elements $X=\{\mathrm{x} 1, \ldots, \mathrm{x} \mathrm{n}\}\left\{\right.$ Idisplaystyle $X=\mid\{\backslash \text { mathbf }\{\mathrm{x}\}\}_{-}\{1\}, \ldots$, Imathbf $\{\mathrm{x}\}$ $\left.\left.{ }_{-}\{\mathrm{n}\} \backslash\right\}\right\} \mathrm{X}=\{\mathrm{x} 1, \ldots \mathrm{xn}\}$ into a collection of $\mathrm{c}$ fuzzy clusters with respect to some given criterion.

Given a finite set of data, the algorithm returns a list of $\mathrm{c}\{$ \displaystyle $\mathrm{c}\} \mathrm{c}$ cluster centers $\mathrm{C}\{\mathrm{c} 1, \ldots \mathrm{Cn}\} \mathrm{C}=\{\mathrm{c} 1, \ldots, \mathrm{c} \mathrm{c}\} \quad\{$ displaystyle $\mathrm{C}=\backslash\{\backslash$ mathbf $\{\mathrm{c}\}$ $\left.\left.\left.{ }_{-}\{1\}, \ldots, \operatorname{lmathbf}\{c\}\right\}_{-}\{\mathrm{c}\} \backslash\right\}\right\}$ and a partition matrix $\mathrm{W}=$ wij in $[0,1] \mathrm{i}=1, \ldots, \mathrm{n} ; \mathrm{j}=1, . . \mathrm{cW}$ $=\mathrm{w} \mathrm{i}, \mathrm{j} \in[0,1], \mathrm{i}=1, \ldots, \mathrm{n}, \mathrm{j}=1, \ldots, \mathrm{c}\left\{\right.$ \displaystyle $\mathrm{W}=\mathrm{w}_{-}\{\mathrm{i}, \mathrm{j}\} \backslash \mathrm{in}$ $[0,1], \backslash ; i=1, \ldots, n, \backslash ; j=1, \ldots, c \mathrm{c}\}$, where each element, w i j \{ldisplaystyle $\left.\mathrm{w}_{-}\{\mathrm{ij}\}\right\}$ wij, tells the degree to which element, $\mathrm{x}$ i $\left\{\right.$ \displaystyle $\backslash$ mathbf $\left.\{\mathrm{x}\} \_\{\mathrm{i}\}\right\}$ xi, belongs to cluster $\mathrm{c} j$ \{ \displaystyle \mathbf $\left.\{\mathrm{c}\} \_\{\mathrm{j}\}\right\} \mathrm{cj}$.

The FCM aims to minimize an objective function:

$$
\begin{aligned}
\underset{c}{\arg \min } \sum_{i=1}^{n} \sum_{j=1}^{c} w_{i j}^{m}|| x i-c j \|^{2} & \text { as } \\
w_{i j} & =\frac{1}{\sum_{k=1}^{c}\left(\frac{x_{i}-c_{j}}{x_{i}-c_{k}}\right)^{\frac{2}{m-1}}}
\end{aligned}
$$

$\mathrm{w} \mathrm{i} \mathrm{j}=1 \sum \mathrm{k}=1 \mathrm{c}(\|\mathrm{x} \mathrm{i}-\mathrm{c} \mathrm{j}\|\|\mathrm{x} \mathrm{i}-\mathrm{ck}\|) 2 \mathrm{~m}-1$. \{ldisplaystyle $\mathrm{w}_{-}\{\mathrm{ij}\}=\{\backslash$ frac $\{1\}\left\{\mid\right.$ sum $\quad\{\mathrm{k}=1\}^{\wedge}\{\mathrm{c}\} \backslash \operatorname{left}(\{\mid$ frac $\quad\{$ left || mathbf $\{\mathrm{x}\} \quad-\{\mathrm{i}\}$-lmathbf $\{\mathrm{c}\}$

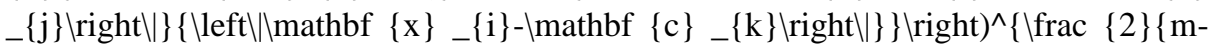
$1\}\}\}\}$.

A probability function is used in the membership function that assigns each local pixel a membership degree value using spatial information inside a square window. This function indicates the probability of the degree of belonging of the pixels adjacent to a category with respect to the central pixel to that category. Eventually new centers and category weights are added to the membership function.

\subsection{Proposed method}

In the chosen method, two types of Flair and T1W brain MRI images were used. Images must first be preprocessed to normalize their pixel location and also to remove additional information such as noise in the images. Both of these images, after preprocessing, go into the fenestration phase to extract the features already mentioned. According to previous information, we know that at the classification stage we have two sections of training and testing of the classification. Training and experimental images were used for this purpose. These images are selected using an algorithm called batch K-cross validation. Finally, the post processing and benchmarking stage is presented. 


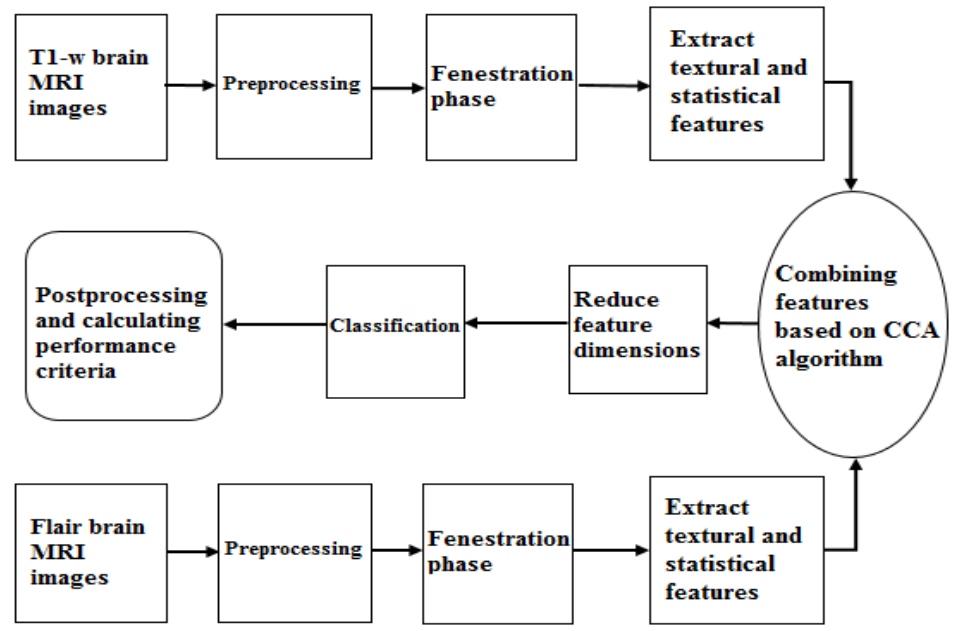

Fig. 3. Method used in the paper

The method performed in this paper (Figure 3) has new features compared to the reference article, including the use of two Canonical Correlation Analysis (CCA) algorithms and also a batch $\mathrm{K}$-cross validation algorithm. The batch K-cross validation algorithm has random values of $\mathrm{K}$ (random) and this process is random (random) and the process $\mathrm{K}$ is repeated to give high reliability of the results.

\section{Results}

The images that are available from our database should first be converted to the common bmp format, the image format in the database is mha*.According to the block diagram in Figure 1, these images are preprocessed to normalize brain MRI images in terms of brightness, pixel location, and size. These images then go into the feature window extraction and extraction phase and extract the desired texture and statistical properties from them. According to the same block diagram, a new method called the CCA algorithm is used to extract new features with better results by combining available features. Due to the large number of these features, we use the Principal Component Analysis (PCA) algorithm to reduce the dimensions of the properties. For classification of tumor in MRI, three classifiers FCM, SVM, PNN are used and also three criteria of sensitivity, specificity and accuracy are used for comparison. The images used in this article include tumor-specific brain MIR images derived from the multifaceted challenge of NCI-MICCAI 2015 tumor classification. These images were in the unknown and were taken from The Cancer Imaging Archive (TCIA) [11]. In this database, tumor-infected areas in brain MRI images are manually identified. The proposed method is implemented for simulated and realistic magnetic resonance images. It should be noted that the target database also contains these (simulated and real) images (Figs 4 and 5). 

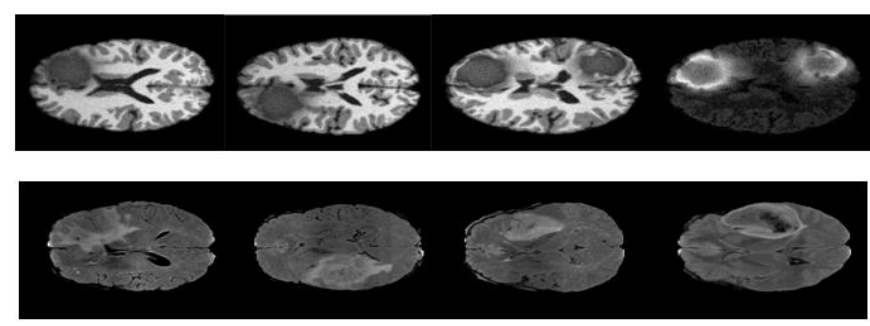

Fig. 4. T1W real and simulated brain MRI images
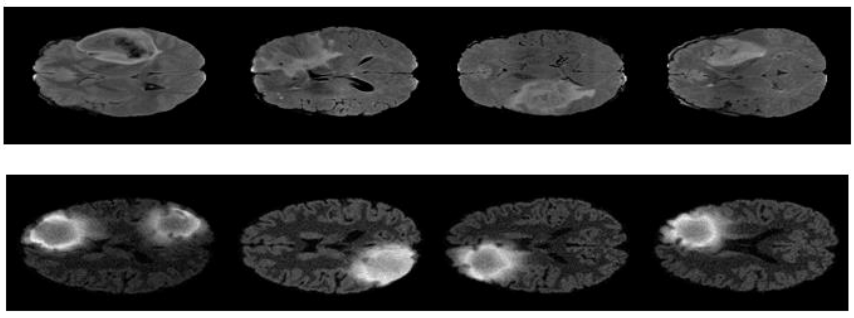

Fig. 5. Flair real and simulated brain MRI images

\subsection{Preprocessing}

Existing images include simulated and real MRI images. In order to apply the preprocessing step, it is important to note that this step is different for each simulated and real MRI image. For simulated images first of all, we first need to convert the images obtained from the database to the current format, so the mha* format is converted to bmp format. Then we need to be able to normalize the pixel location of MRI images. To do this, we convert the image size to $200 \times 200$ pixels to eliminate this extra pixel. For the preprocessing of real images, in addition to the steps performed for simulated images, two additional steps are required, including normalization of the brightness intensity and applying the histogram matching algorithm. The basis for normalizing the intensity of the illumination is that the standard deviation of the MRI image columns is calculated and then the pixels that each column has are subdivided into the corresponding standard deviation. In the histogram algorithm, an image of a set of real images is considered as a reference image, then the reference image histogram and other real images are computed and matched. In Figure 6, the real MRI image and also its calculated histogram is shown in graphs. Figure 7 also shows the real MRI image with its histogram before applying the histogram matching algorithm and after applying the algorithm. 

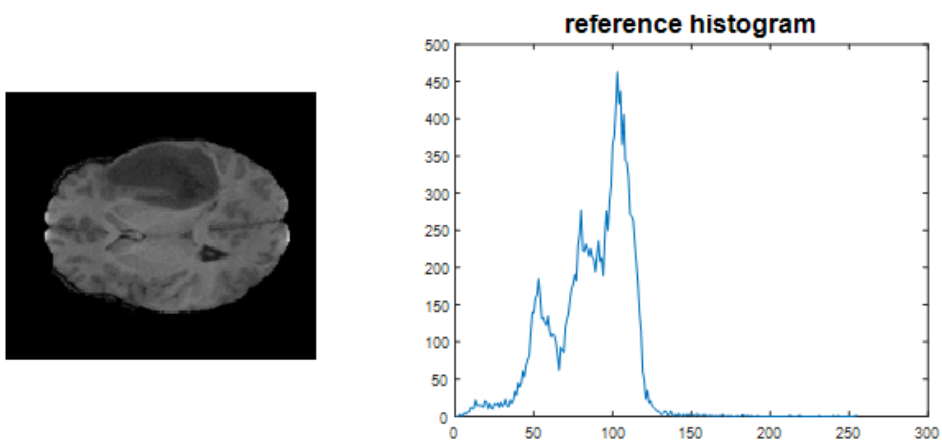

Fig. 6. Reference brain MRI images and its histogram
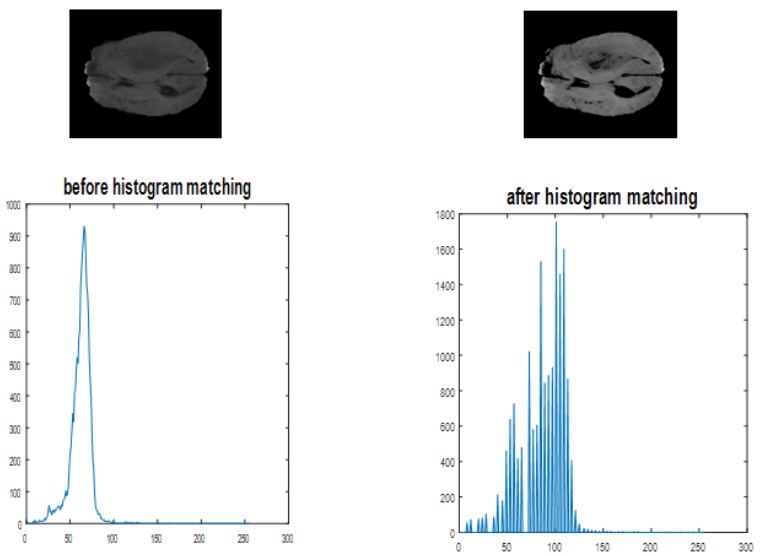

Fig. 7. Real brain MRI image, before and after applying the algorithm along with the histogram

\subsection{Fenestration}

Fenestration is divided into two types of overlapping and non-overlapping. This article uses overlapping fenestration. The overlapping mode of fenestration works by sampling the pixels in two rows and columns of magnetic resonance images. Around each of these pixels, we place a $10 \times 10$ window to extract the statistical properties and transform and store them in vector. By doing this, we conclude that for each magnetic resonance imaging, 4096 of $10 \times 10$ windows are formed (Figure 8 ): 
sub-sampling pixels

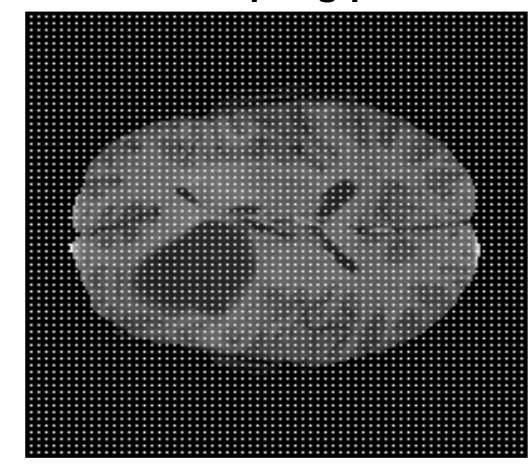

Fig. 8. Overlapping fenestration around the pixels centered on the $10 \times 10$ windows

At the fenestration stage, the features extracted from these windows include 315 numbers. Of these, 32 attributes are assigned to the oriented gradient histogram, 20 are attributes of the gray surface row matrix, 256 are attributes of linear binary pattern attributes, and the remaining 7 attributes belong to first-order statistics. Therefore, it can be concluded that from each window a feature vector of $315 \times 1$ is obtained. Also, each brain MRI image has 4096 of $10 \times 10$ windows. Consequently, for each brain MRI image, we will have a feature matrix with a dimension of $315 \times 4096$.

\subsection{Dimensionality matrix dimension reduction}

This part of the classification system consists of two steps:

The first step is to use the PCA algorithm which reduces the number of features. The basis of the kettle is that a mathematical method (orthogonal transformation) is used to convert a set of correlated variables into a set of closed variables. In most cases, $20 \%$ of the primary dimensions of the output matrix of this algorithm have a different view of the feature matrix, which has about $99 \%$ of the matrix changes of the primary attribute.

The second step is to remove the pixels on the margins of the brain MRI images. In brain MRI images, we usually come across black pixels around the image, or pixels that have zero values. To remove this part of the images, you should set it to zero if fenestration is zero, as a result, the entire pixel becomes zero. Removing these pixels from the margins of the brain MRI images will cause no additional processing, reducing the feature matrix dimension or the same number of samples.

\subsection{Classification}

In this paper we use three classifiers FCM, SVM, PNN. This stage also consists of two parts. Training and testing are two parts to consider in classifying. In the training section, the classifier of a training matrix containing multiple brain MRI image features is categorized as input. In the experiment section, the features of the images not 
used in the previous section are categorized as predictors. The results obtained from the classification are evaluated with the criteria of sensitivity, specificity and accuracy. Sensitivity (true positive rate) means the proportion of positive items that the test correctly marks as positive. Diagnosis (true negative rate) means the ratio of negatives that the test correctly marks as negative. The following are the relationships to these three criteria:

$$
\begin{aligned}
& \text { sensitivity }=\frac{n_{T P}}{n_{T P}+n_{F N}} 100 \% \\
& \text { specificity }=\frac{n_{T N}}{n_{T N}+n_{F P}} 100 \% \\
& \text { accuracy }=\frac{n_{T P}+n_{T N}}{n_{T P}+n_{T N}+n_{F P}+n_{F N}} 100 \%
\end{aligned}
$$

True Positive (TP): The sample is a member of a positive category and is recognized as a member of the same class; False Negative (FN): The sample is a member of the positive class and is recognized as a member of the negative class; True Negative (TN): The sample is a member of a negative class and is recognized as a member of the same class; False Positive (FP): The sample is a member of the negative class and is recognized as a positive class member.

\subsection{Database and simulated parameters}

The brain MRI images used in this study were taken from the TCIA. In total, 72 brain MRI images were used from the database, 36 of which included real brain MRI images and 36 of the simulated brain MRI images. Since the purpose of this paper is to increase the reliability of the results obtained from the classification, the batch $\mathrm{K}$ validation algorithm is used. In this method, the brain MRI images are divided into $\mathrm{K}$ groups of different independent groups. It should be noted that the number of images in each group is the same and the segmentation is done at random. To classify these images, the (K-1) group is assigned to training images and only one group contains experimental images. This process is repeated $\mathrm{K}$ for the classifier, then the average of the three criteria is sensitivity, specificity and accuracy. The results can be a high reliability result. The PCA algorithm performs feature dimensionality reduction and is trained in classification. For the experimental group, the statistical properties of this group are extracted and after applying the PCA algorithm the predictor is classified as input. Finally, the output from the classifier consists of two types of labels, whether tumor or healthy. 


\subsection{Simulation results and comparison with previous results}

To compare the results of the classification of brain MRI images, first the actual brain MRI images and then the results of the simulated MRI images are presented. The focal correlation algorithm is used to improve the performance of the proposed method. This algorithm integrates information from two types of brain MRI images. By doing so, the said three criteria will have an acceptable increase. Table 1 shows the results of the three FCM, SVM, and PNN classifiers. In the proposed method for $\mathrm{K}=2$, which actually represents two groups of $18 \mathrm{~T} 1 \mathrm{~W}$ real brain MRI images, as training images and another group of 18 real brain MRI images as experimental images.

Table 1. Results from tumor classification for real brain MRI images for $\mathrm{K}=2$

\begin{tabular}{|c|c|c|c|c|c|}
\hline \multicolumn{1}{|c|}{ Classification } & FCM & SVM & PNN & Reference [11] & Reference [12] \\
\cline { 1 - 5 } Percentage of sensitivity & 72.2 & 70.0 & 86.6 & ---- & 84 \\
\hline Percentage of specificity & 95.1 & 94.0 & 94.1 & ---- & 91 \\
\hline Percentage of accuracy & 95.0 & 93.8 & 94.0 & 93.1 & \\
\hline
\end{tabular}

Because of the choice of $\mathrm{K}=2$, we find that the process is repeated twice, and the percentages calculated for each criterion in the performance evaluation of the proposed method are twice the average performance of the process. At each time of the process, one group includes the training brain MRI images and the other the experimental group. Since we have to use the multiple of $72(\mathrm{~K}=2,6,9)$ for the selection. This process will be repeated 3 times and the training and experimental groups will be moved once the process is executed.

Table 2 shows the results for $\mathrm{K}=6$ and for the other $\mathrm{K} 9$ the results are shown in Tables 3:

Table 2. Results from tumor classification for real brain MRI images for $K=6$

\begin{tabular}{|l|c|c|c|c|c|}
\hline \multicolumn{1}{|c|}{ Classification } & FCM & SVM & PNN & Reference [11] & Reference [12] \\
\cline { 1 - 5 } Percentage of sensitivity & 71.2 & 71.2 & 71.2 & ---- & 71 \\
\hline Percentage of specificity & 95.1 & 94.0 & 94.2 & ---- & 90.4 \\
\hline Percentage of accuracy & 94.9 & 93.9 & 94.1 & 93.1 & ---- \\
\hline
\end{tabular}

Table 3. Results from tumor classification for real brain MRI images for $K=9$

\begin{tabular}{|c|c|c|c|c|c|}
\hline Classification & \multirow{2}{*}{ FCM } & \multirow{2}{*}{ SVM } & \multirow{2}{*}{ PNN } & \multirow{2}{*}{ Reference [11] } & \multirow{2}{*}{ Reference [12] } \\
\hline Criterion & & & & & \\
\hline Percentage of sensitivity & 75.9 & 73.5 & 69.6 & $\begin{array}{ll}--- \\
--\end{array}$ & 67 \\
\hline Percentage of specificity & 94.1 & 94.3 & 94.2 & ---- & 90.9 \\
\hline Percentage of accuracy & 94.4 & 94.0 & 94.0 & 93.1 & ---- \\
\hline
\end{tabular}


The results for simulated brain MRI images were also performed for different Ks according to the previous procedure. These results are shown in Tables 4 to 6 .

Table 4. Results from tumor classification for simulated brain MRI images for $\mathrm{K}=2$

\begin{tabular}{|c|c|c|c|c|c|}
\hline Classification & \multirow{2}{*}{ FCM } & \multirow{2}{*}{ SVM } & \multirow{2}{*}{ PNN } & \multirow{2}{*}{ Reference [11] } & \multirow{2}{*}{ Reference [12] } \\
\hline Criterion & & & & & \\
\hline Percentage of sensitivity & 97.1 & 97.8 & 97.2 & 96.2 & 97.1 \\
\hline Percentage of specificity & 96.3 & 97.5 & 97.6 & 95.7 & 91.5 \\
\hline Percentage of accuracy & 96.4 & 97.6 & 97.5 & 96.7 & $\begin{array}{ll}--- \\
---\end{array}$ \\
\hline
\end{tabular}

Table 5. Results from tumor classification for simulated brain MRI images for $\mathrm{K}=6$

\begin{tabular}{|c|c|c|c|c|c|}
\hline Classification & \multirow{2}{*}{ FCM } & \multirow{2}{*}{ SVM } & \multirow{2}{*}{ PNN } & \multirow{2}{*}{ Reference [11] } & \multirow{2}{*}{ Reference [12] } \\
\hline Criterion & & & & & \\
\hline \begin{tabular}{|l|} 
Percentage of sensitivity \\
\end{tabular} & 97.5 & 97.7 & 96.9 & 96.2 & 97.1 \\
\hline Percentage of specificity & 96.5 & 97.4 & 97.5 & 95.7 & 92 \\
\hline Percentage of accuracy & 96.5 & 97.6 & 97.4 & 96.7 & ---- \\
\hline
\end{tabular}

Table 6. Results from tumor classification for simulated brain MRI images for K=9

\begin{tabular}{|c|c|c|c|c|c|}
\hline Classification & \multirow{2}{*}{ FCM } & \multirow{2}{*}{ SVM } & \multirow{2}{*}{ PNN } & \multirow{2}{*}{ Reference [11] } & \multirow{2}{*}{ Reference [12] } \\
\hline Criterion & & & & & \\
\hline Percentage of sensitivity & 72.2 & 70.0 & 68.6 & ---- & 72.1 \\
\hline Percentage of specificity & 95.1 & 94.0 & 94.1 & ---- & 91.5 \\
\hline Percentage of accuracy & 95.0 & 93.8 & 94.0 & 93.1 & ---- \\
\hline
\end{tabular}

\subsection{Integrate extracted features to increase accuracy}

Brain MRI images used for classification have various types such as T1W, T1C, T2C, T2W, Flair. In this way, the information contained in these images is merged. This increases the accuracy of the classifier and increases its speed. Among the types of brain MRI images, we have analyzed and analyzed T1W and Flair images. The CCA algorithm is used to combine the features of these two images. The dynamic range of the extracted values is different due to the variety of extracted features, which is a reason for the imbalance in the feature matrix. For this purpose, we normalize the dynamic range until these differences are eliminated or minimized. The procedure is that in each column of the attribute matrix, the average value of the column is subtracted from the corresponding column and then subtracted from the standard deviation of that column. By doing so, the average value of all columns will be zero and their variance will be equal to one. In (6) the normalized properties are shown. This relationship is performed to extract features from training and experimental MRI images of the brain.

$$
y_{i}=\frac{x_{i}-\bar{x}_{i}}{\operatorname{std}_{i}}
$$


$y_{i}$ : Normalized Features; ${ }^{x_{i}}$ : Not normalized features $\bar{x}_{i}$ : Average of each attribute matrix column; ${ }^{s t d_{i}}$ : Standard deviation of each feature matrix column

We now use the PCA algorithm to reduce the size of the extracted features. Since two types of T1W and Flair brain MRI images were used in this study, we have two feature matrices for T1W and Flair brain MRI images as well as two matrices for the experimental group. The CCA algorithm is used to integrate these features and to have an experimental and educational feature matrix. For classification, the matrix of training and experimental features is also given to the classifier to identify existing pixel tags. The results of the above steps are shown in the table first for real images and then for simulated images.

Table 7. Results from classification of two types of real brain MRI images using CCA algorithm for $\mathrm{K}=2$

\begin{tabular}{|c|c|c|c|c|c|}
\hline Classification & \multirow{2}{*}{ FCM } & \multirow{2}{*}{ SVM } & \multirow{2}{*}{ PNN } & \multirow{2}{*}{ Reference [11] } & \multirow{2}{*}{ Reference [12] } \\
\hline Criterion & & & & & \\
\hline Percentage of sensitivity & 91.5 & 91.7 & 92.6 & ---- & 90.1 \\
\hline Percentage of specificity & 94.6 & 94.7 & 94.1 & $\begin{array}{ll}--- \\
---\end{array}$ & 93 \\
\hline Percentage of accuracy & 94.5 & 94.6 & 94.1 & 93.1 & ---- \\
\hline
\end{tabular}

Table 8. Results from classification of two types of real brain MRI images using CCA algorithm for $\mathrm{K}=6$

\begin{tabular}{|c|c|c|c|c|c|}
\hline Classification & \multirow{2}{*}{ FCM } & \multirow{2}{*}{ SVM } & \multirow{2}{*}{ PNN } & \multirow{2}{*}{ Reference [11] } & \multirow{2}{*}{ Reference [12] } \\
\hline Criterion & & & & & \\
\hline Percentage of sensitivity & 93.1 & 91.0 & 90.9 & ---- & 90.4 \\
\hline Percentage of specificity & 93.8 & 94.6 & 94.1 & ---- & 94.1 \\
\hline Percentage of accuracy & 94.6 & 94.6 & 94.1 & 93.1 & ---- \\
\hline
\end{tabular}

Table 9. Results from classification of two types of real brain MRI images using CCA algorithm for $\mathrm{K}=9$

\begin{tabular}{|l|c|c|c|c|c|}
\hline \multicolumn{1}{c|}{ Classification } & \multirow{2}{*}{ FCM } & SVM & PNN & Referion &
\end{tabular}

Now, we integrate the features of simulated brain MRI images using the focal correlation analysis algorithm and show the results for different Ks. 
Table 10. Results from classification of two types of real brain MRI images using CCA algorithm for $\mathrm{K}=2$

\begin{tabular}{|c|c|c|c|c|c|}
\hline Classification & \multirow{2}{*}{ FCM } & \multirow{2}{*}{ SVM } & \multirow{2}{*}{ PNN } & \multirow{2}{*}{ Reference [11] } & \multirow{2}{*}{ Reference [12] } \\
\hline Criterion & & & & & \\
\hline Percentage of sensitivity & 98.9 & 98.6 & 98.2 & 96.2 & 97.1 \\
\hline Percentage of specificity & 98.5 & 97.6 & 95.7 & 95.7 & 96.5 \\
\hline Percentage of accuracy & 98.7 & 97.6 & 96.7 & 96.7 & $\begin{array}{ll}--- \\
---\end{array}$ \\
\hline
\end{tabular}

Table 11. Results from classification of two types of real brain MRI images using CCA algorithm for $\mathrm{K}=6$

\begin{tabular}{|c|c|c|c|c|c|}
\hline Classification & \multirow{2}{*}{ FCM } & \multirow{2}{*}{ SVM } & \multirow{2}{*}{ PNN } & \multirow{2}{*}{ Reference [11] } & \multirow{2}{*}{ Reference [12] } \\
\hline Criterion & & & & & \\
\hline Percentage of sensitivity & 98.9 & 98.9 & 98.5 & 96.2 & 98.8 \\
\hline Percentage of specificity & 98.1 & 97.7 & 98.0 & 95.7 & 96.6 \\
\hline Percentage of accuracy & 98.1 & 97.7 & 98.0 & 96.7 & ---- \\
\hline
\end{tabular}

Table 12. Results from classification of two types of real brain MRI images using CCA algorithm for $\mathrm{K}=9$

\begin{tabular}{|c|c|c|c|c|c|}
\hline Classification method & \multirow{2}{*}{ FCM } & \multirow{2}{*}{ SVM } & \multirow{2}{*}{ PNN } & \multirow{2}{*}{ Reference [11] } & \multirow{2}{*}{ Reference [12] } \\
\hline Criterion & & & & & \\
\hline Percentage of sensitivity & 99.5 & 99.0 & 99.0 & 96.2 & 99.1 \\
\hline Percentage of specificity & 98.5 & 97.8 & 97.8 & 95.7 & 96.9 \\
\hline Percentage of accuracy & 98.8 & 97.8 & 97.8 & 96.7 & --- \\
\hline
\end{tabular}

Figure 7 shows a comparison chart of sensitivity criteria before and after applying focal correlation analysis algorithm for brain tumor classification in simulated brain MRI images based on different $\mathrm{k}$ values in batch $\mathrm{k}$-cross validation algorithm, using FCM classifier. According to the number of simulated brain MRI images (36), if $\mathrm{k}=$ 2, 18 images are selected as training images and 18 images as experimental images and the number of repeats is 2 . If $\mathrm{k}=6,30$ images are selected as training images and 6 images as experimental images and the number of iterations is 6 . If $\mathrm{k}=9,32$ images are selected as training images and 4 images as experimental images and the number of iterations is 9 .

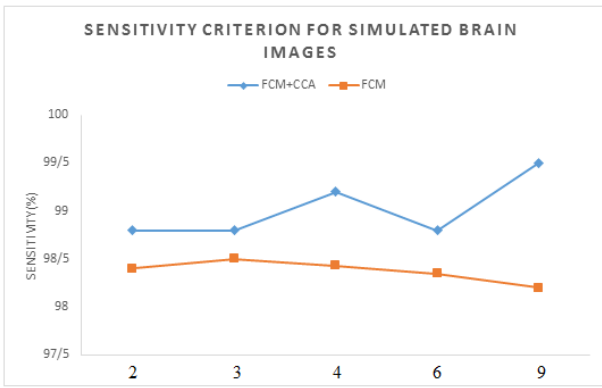

Fig. 9. Applying CCA algorithm for simulated images on sensitivity criteria for FCM 
As shown in Figure 7, in the FCM classifier, applying the CCA algorithm to simulated brain MRI images increased the sensitivity criterion by $0.5 \%$ to $1.5 \%$, it means that the rate of detection of tumor pixels has increased. Figure 8 shows comparison charts of sensitivity criteria before and after applying the focal correlation analysis algorithm for classification of brain tumor in real brain MRI images based on different $\mathrm{k}$ values in batch cross validation algorithm using FCM classifier. The number of actual brain MRI images used is 36 as before. In this experiment, $\mathrm{k}=2, \mathrm{k}=6$ and $\mathrm{k}=$ 9 has been chosen. Tumor classification results show that the sensitivity criterion, especially for real brain MRI images, is increased using the CCA algorithm applied at the feature extraction stage.

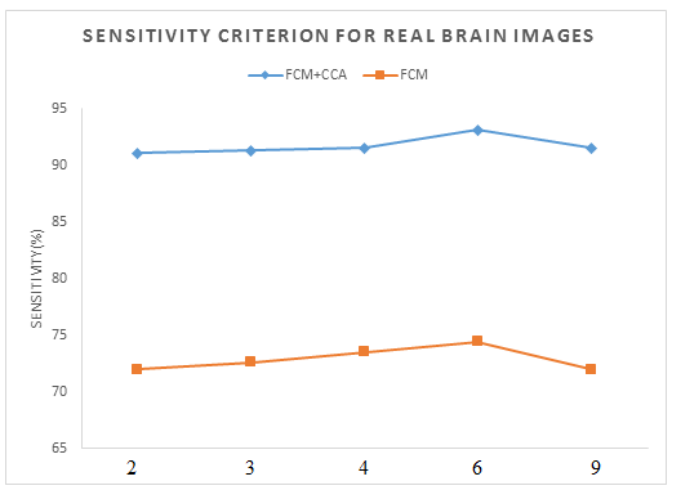

Fig. 10.Comparison chart of sensitivity criteria for FCM classifier before and after applying CCA algorithm to real images

As shown in Figure 8, for real brain MRI images in any FCM classifier, the sensitivity criterion increase is more noticeable. Applying the CCA algorithm increased the sensitivity criterion by approximately $20 \%$. In the following, we evaluate the three criteria of sensitivity, specificity, and accuracy for the FCM classifier between the proposed and the reference method [11, 12]. The total number of images used in the reference [12 and 11] to classify tumors in brain MRI images are 25 and 35, but in this article a total of 72 images are used, so the database used in this article is larger than the reference database [11 and 12].

In reference [11], for real brain MRI images only the accuracy criterion is mentioned and two sensitivity and specificity criteria have not been reported. In this paper, only the best results from the reference [11 and 12] to the SVM classifier are mentioned and the results that are lower than the best results are avoided. Also, in reference [12], the sensitivity and specificity with dice score coefficient are considered.

In reference [12] to increase reliability, the k-batch cross validation algorithm is only used for $\mathrm{k}=10$, ie $90 \%$ of the data is used as training images and only $10 \%$ of the data is used as experimental images. But in this paper, different values of $\mathrm{k}=2, \mathrm{k}$ $=6$ and $\mathrm{k}=9$ are selected which use $50 \%, 33 \%, 25 \%, 16 \%$ and $11 \%$ of the data as 
experimental images, respectively. This makes the proposed method more reliable than the reference.

Figure 9 is plotted to compare the sensitivity criteria for classification of simulated brain MRI images between the proposed and the reference [11] method. Because sensitivity and specificity criteria are not reported in the reference [11] for classification of real brain MRI images, only the diagrams of the proposed method in this paper using FCM, PNN and SVM classifiers for images The actual brain MRI is shown in Figure 10 for the accuracy criterion.

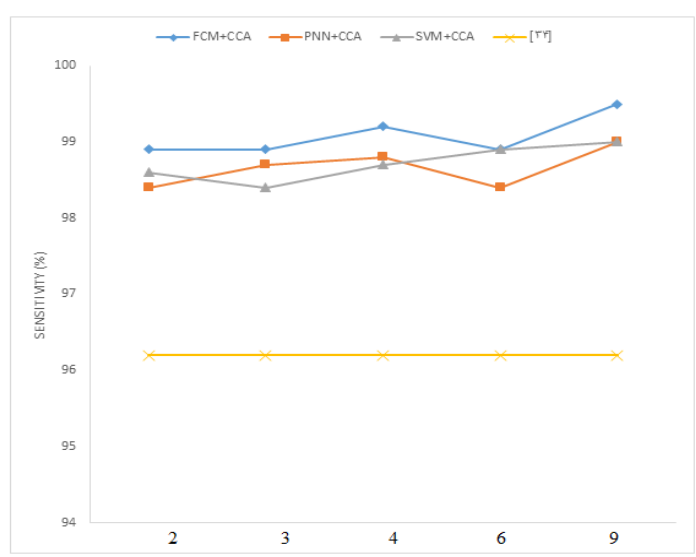

Fig. 11.Sensitivity criterion classification of simulated brain MRI images

As can be seen in Figure 9, the sensitivity criterion in simulated brain MRI images after applying the CCA algorithm increased by $2 \%$ over reference [11] that this increase is due to the number of different batches in the $\mathrm{k}$ batch cross validation algorithm. As is illustrated in this figure, the use of FCM classifiers performs better than other classifiers, which in this paper focuses more on the use of this classifier in the proposed CCA structure.

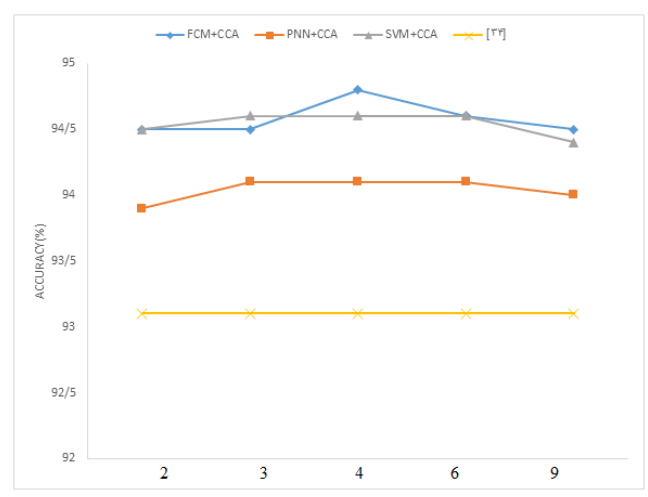

Fig. 12. Classification accuracy criteria of real brain MRI images in the proposed method for three classifiers 
As can be seen in Figure 10, the accuracy criterion in real brain MRI images after applying the CCA algorithm increased by approximately $1.5 \%$ relative to the reference [11]. Also, by accurately measuring the accuracy of actual images and comparing the three proposed methods for classification with the reference article method [11], we conclude that using the CCA structure improves the performance of the system substantially. Also, by comparing the three methods proposed in this paper for classification, we find that the use of FCM classifiers performs better than the SVM and PNN classifiers and improves system performance.

\section{Conclusion}

In this paper, a method for classifying tumors in simulated and real brain MRI images using statistical features is presented. This method reduces the complexity and reduces the time and memory needed to execute the classification process. The method presented in this article is a fully automated one. In this method, sensitivity, specificity and accuracy criteria for simulated brain MRI images using different classifiers are obtained which compared to the references [11 and 12] and other available methods demonstrate the effectiveness of the proposed method. Another advantage of the proposed method in this paper is the high reliability due to the use of $\mathrm{k}$ batch cross validation algorithm with different batch number, it means that the reported numbers for the three criteria of sensitivity, specificity, and accuracy are equal to the mean of these criteria at $\mathrm{k}$ times the unique iteration of the classification process. At the fenestration stage, the number of windows due to the small size of the sample windows and their overlap is large, which can be a drawback to the proposed method.

\section{$5 \quad$ References}

[1] Florian Roser MD, Jürgen Honegger MD, Martin U. Schuhmann MD Marcos S.Tatagiba MD, Meningiomas, (2012) Nerve Sheath Tumors, and Pituitary Tumors: Diagnosis and Treatment, Hematology/Oncology Clinics of North America, 26, 4, 855-879. https://doi. org/10.1016/j.hoc.2012.04.005

[2] Jamshidi-Naeini, Y., Bavil, A. K., Egal, A., \& Oldewage-Theron, W. (2019). Hemoglobin and ferritin concentrations are positively associated with blood pressure and hypertension risk in older adults: a retrospective cross-sectional study, Sharpeville, South Africa. Asia Pac J Clin Nutr, 28(3), 533-543.

[3] Almasi M., (2016), New method based on Image processing to enhanced the Accuracy and Precision Of table tennis player's performance, Journal of Research in Science, Engineering, and Technology, Vol4, No.03, 1-3, https://doi.org/10.24200/jrset.vol4iss03pp1-3

[4] D. Saraswathi, G. Sharmila, E. Srinivasan, (2018), An Automated Diagnosis system using Wavelet based SFTA Texture Features", IEEE, International Conference on Information Communication and Embedded Systems (ICICES), pp.1-5, Chennai-India. https://doi.org/ 10.1109/icices.2014.7034123

[5] N. Nabizadeh, M. Dorodchi, M. Kubat, (2016), Automatic tumor lesion detection and segmentation using modified winnow algorithm', IEEE, International Symposium on Bi- 
omedical Imaging (ISBI), pp. 71-74, NewYork-USA. https://doi.org/10.1109/isbi.2015.7 $\underline{163819}$

[6] S. Kumar Adhikari, J. K. Sing, D. Kumar Basu, M. Nasipuri, (2015), A spatial fuzzy CMeans algorithm with application to MRI image segmentation', IEEE, Eighth International Conference on Advances in Pattern Recognition (ICAPR), pp. 1-6, Kolkata-India. https://doi.org/10.1109/icapr.2015.7050691

[7] K. Verma, S. Urooj, Rituvijay, (2014). Effective Evaluation of Tumour Region in Brain MR Images Using Hybrid Segmentation', IEEE, International Conference on Computing for Sustainable Global Development (INDIACom), pp. 570-574, New Delhi-India. https:// doi.org/10.1109/indiacom.2014.6828024

[8] N. Nabizadeh, (2015), Automated Brain Lesion Detection and Segmentation Using Magnetic Resonance Images", Ph.D. Thesis, University of Miami.

[9] W. Chen, R. Smith, N. Nabizadeh, K. Ward, C. Cockrell, J. Ha, (2020), Texture analysis of brain CT scans for ICP prediction', SPRINGER, image and signal processing, Vol. 6134, pp. 568-575. https://doi.org/10.1007/978-3-642-13681-8_66

[10] V. Amsaveni, N. A. Singh, (2014), Detection of Brain Tumor using Neural Network", IEEE, Fourth International Conference on Computing, Communications and Networking Technologies (ICCCNT), pp. 1-5, Tamil Nadu-India. https://doi.org/10.1109/iccent.2013. $\underline{6726524}$

[11] N. Nabizadeh, M. Kubat, (2015). Brain tumors detection and segmentation in MR images: Gabor wavelet vs. statistical features", Computers and Electrical Engineering, Vol. 45, pp.286-301. https://doi.org/10.1016/j.compeleceng.2015.02.007

[12] Sajid, S., Hussain, S. \& Sarwar, A. Brain Tumor Detection and Segmentation in MR Images Using Deep Learning. Arab J Sci Eng 44, 9249-9261 (2019). https://doi.org/10.10 07/s13369-019-03967-8.

[13] https://www.smir.ch/BRATS/Start2015

\section{Authors}

Hamed Samadi Goushchi is MSc in Electrical engineering from Faculty of Electrical Engineering, Urmia university of Technology. He has participated as in international conferences including the Image processing, Electrical Units and Detection systems.

Yaghoub Pourasad is an assistant professor in electrical engineering, from Faculty of Electrical Engineering, Urmia university of Technology. He works also as a visiting professor at the Universities of Amirkabir University which has the organizer of the international conferences in the electrical engineering and biomedical scopes. His main interests are image processing and electrical unit's usage in biomedical science which has published more than 10 papers in this subject. Email: y.pourasad@uut.ac.ir; alireza.e.amiri@gmail.com

Article submitted 2020-07-09. Resubmitted 2020-08-07. Final acceptance 2020-08-08. Final version published as submitted by the authors. 